\title{
ESTRUTURA E COMPOSIÇÃO FLORÍSTICA DE UM FRAGMENTO DE FLORESTA semidecídua na região agreste do estado da Paraíba, Nordeste BRASILEIRO
}

\author{
Anderson Silva Pinto ${ }^{1 *}$, Fernanda Kalina da Silva Monteiro ${ }^{2}$, Sérgio de Faria Lopes ${ }^{3}$
}

\author{
${ }^{1}$ Programa de Pós-graduação em Etnobiologia e Conservação da Natureza, Departamento de Biologia, Universidade Federal Rural de Pernambuco, 52171 - \\ 900, Recife, Brasil. \\ ${ }^{2}$ Programa de Pós-graduação em Botânica, Departamento de Biologia, Universidade Federal Rural de Pernambuco, 52171-900, Recife, Brasil. \\ ${ }^{3}$ Departamento de Biologia, Universidade Estadual da Paraíba, 58429-500, Campina Grande, Brasil. \\ *Autor para correspondência: anderson.slvp@gmail.com
}

Recebido em 23 de agosto de 2019. Aceito em 17 de dezembro de 2019. Publicado em 30 de dezembro de 2019.

Resumo - Na região Nordeste as florestas semidecíduas estão sendo substituídas por grandes lavouras e pequenas plantações. Nesse sentido, levantamentos fitossociológicos se fazem importantes por abordar aspectos ligados a estrutura, composição e o estado de conservação dos fragmentos remanescentes ameaçados. Assim objetivou-se descrever a estrutura e composição florística da comunidade arbórea de um fragmento de floresta estacional semidecidual no município de Lagoa Seca, Paraíba, Brasil. Para isso, foram alocadas 25 parcelas permanentes com dimensões de $400 \mathrm{~m}^{2}$, sendo todos os indivíduos com circunferência à altura do peito $\geq 15$ centímetros aferidos. A partir dos dados coletados foram elaboradas listas florísticas, fitossociológicas, gráficos de distribuição de classes de diâmetro e altura, índice de diversidade de Shannon (H’) e área basal total da comunidade. Foram amostrados 1835 indivíduos, pertencentes a 76 espécies e 27 famílias botânicas. O índice de Shannon $(3,61)$ foi considerado alto enquanto o valor de área basal total $\left(23,99 \mathrm{~m}^{2} / \mathrm{ha}\right.$ ) foi considerado baixo quando comparados a outros fragmentos semelhantes. Apesar da alta diversidade, o baixo valor de área basal pode estar relacionado aos fatores antrópicos, uma vez que foi percebido que o corte seletivo é constante na área, podendo ser este o mesmo cenário vivenciado por outras áreas ainda não amostradas.

Palavras-chave: Fitossociologia; Diversidade; Ecologia Vegetal.

\section{Structure and floristic composition of a semideciduous fragment forest in agreste region of Paraíba state,} Brazilian Northeast

Aвstract - Semideciduous forests in Northeast region are being substituted by large crops and small plantations. In this sense, phytosociological surveys are important to treat aspects related to the structure, composition and conservation status of the remaining threatened fragments. The aim of this study was to describe the structure and floristic composition of the tree community of a semideciduous seasonal forest fragment in the municipality of Lagoa Seca, Paraíba, Brazil. For this purpose, the permanent plots method was applied, using 25 plots with dimensions of $400 \mathrm{~m}^{2}$ and all individuals with circumference at breast height $>15$ were measured. From the collected data floristic phytosociological lists, distribution charts of diameter and height classes, Shannon diversity index $\left(\mathrm{H}^{\prime}\right)$ and total basal area of the community from the collected data. The fragment sampled presented 1.835 individuals belonging 76 species and 27 botanical families. Shannon's index (3.61) was considered high while the total basal area value $\left(23.99 \mathrm{~m}^{2} / \mathrm{ha}\right.$ ) was considered low when compared to other similar fragments. Despite the high diversity, the low value of basal area may be related to anthropogenic factors, since it was noticed that selective cutting is constant in the area, which may be the same scenario experienced by other areas not sampled yet. 
KeYwords: Phytosociology; Diversity; Plant Ecology.

ESTRUCTURA Y COMPOSICION FLORISTICA DE UN FRAGMENTO DE BOSQUE SEMIDECIDUO EN LA REGIÓN AGRESTE DEL ESTADO de Paraiba, Nordeste de Brasil

RESUMEN - Se están reemplazando los bosques semideciduos en la región Nordeste de Brasil por grandes cultivos y pequeñas plantaciones. En este sentido, los estudios fitosociológicos son importantes para abordar aspectos relacionados con la estructura, composición y conservación de los fragmentos amenazados. Así, el propósito de este estudio es describir la estructura y la composición florística de la comunidad arbórea de un fragmento de bosque estacional semideciduo en el municipio de Lagoa Seca, Paraíba, Brasil. Para ello, se asignaron 25 parcelas permanentes con dimensiones de $400 \mathrm{~m}^{2}$, siendo todos los individuos con circunferencia a la altura del pecho $>15$ centímetros medidos. A partir de los datos recopilados, se desarrollaron listas florísticas, fitosociológicas, gráficos de distribución de clases de diámetro y altura, índice de diversidad de Shannon (H ) y área basal total de la comunidad. Fueron muestreados 1835 individuos de 76 especies y 27 familias botánicas. El índice de Shannon $(3,61)$ se consideró alto, mientras que el área basal total $(23,99$ m²/ha) se consideró baja en comparación con otros fragmentos similares. A pesar de la gran diversidad, el área basal baja puede estar relacionado con factores antrópicos, ya que se observó que el corte selectivo es constante en el área, que puede ser el mismo escenario experimentado por otras áreas aún no muestreadas.

Palabras clave: Fitosociología; Diversidad; Ecología Vegetal.

\section{INTRODUÇÃO}

Aproximadamente $40 \%$ das áreas tropicais e subtropicais do planeta são cobertas por florestas fechadas ou abertas (Murphy e Lugo 1986). As florestas fechadas ou úmidas ocorrem em regiões que não apresentam sazonalidade climática e apresentam precipitação maior que $2.000 \mathrm{~mm}$ (Pennington et al. 2018). À medida que o regime de chuvas diminui e a sazonalidade climática aumenta as florestas úmidas vão dando espaço às savanas e florestas secas (Dexter et al. 2018; Pennington et al. 2018).

As florestas secas ocorrem na Austrália, Sul da Ásia, África e grande parte da América do Sul (Janzen 1988), tendo como característica principal a presença de espécies lenhosas que perdem suas folhas durante a estação seca (decíduas) (Pennington et al. 2000). Na América Latina, as florestas secas ocorrem em solos férteis e pouco ácidos, que são características fundamentais para diferenciá-las das savanas (Miranda et al. 2018; Pennington et al. 2018).

É comum observar a presença de transições entre as florestas úmidas e florestas secas/savanas (Dexter et al. 2018; Pennington et al. 2018), nessas localidades geralmente são encontradas as florestas semidecíduas, vegetação caracterizada por suas árvores (até 50\%) perderem suas folhas em determinada época do ano (IBGE 2012). No Brasil, essas são transições gradativas entre as florestas úmidas que se encontram ao longo da costa e outras formações vegetais localizadas no interior do país (Oliveira-Filho e Ratter 1995; Rodal et al. 2008). Um exemplo nítido desse cenário é expresso na região Nordeste, em que as florestas semidecíduas formam uma transição marcante entre a vegetação típica da zona da mata no litoral e da zona do sertão que é caracterizada pela Caatinga semiárida (Rodal et al. 2005; IBGE 2012). No entanto, é importante chamar atenção para o fato de que estas florestas transicionais podem estar subamostradas na região Nordeste. Essa tendência pode ser vista no estudo de Miranda et al. (2018), em que os autores apontam a existência de 115 levantamentos de florestas transicionais entre floresta atlântica (floresta úmida) e cerrado (savana) e 49 levantamentos de florestas transicionais entre floresta atlântica e caatinga (floresta seca).

Além do baixo número de levantamentos, essas florestas sofrem com diminuição de suas áreas por conta da sobre-exploração (Tabarelli e Santos 2004). De forma contínua, as florestas semidecíduas, principalmente 
aquelas chamadas regionalmente de brejos de altitude, têm sido substituídas por grandes lavouras e pequenas culturas de subsistência (Lins 1989).

Nesse sentido, levantamentos fitossociológicos podem contribuir com o aumento de informações acerca das florestas semidecíduas no Nordeste, pois além de trazer informações sobre a composição de espécies, esses levantamentos também abordam aspectos ligados à estrutura, dinâmica, relações ecológicas e atual estado de conservação das comunidades estudadas.

Entender os aspectos ligados a ecologia das espécies vegetais também pode contribuir para uma maior compreensão dos serviços ecossistêmicos oferecidos às populações locais. Os serviços ecossistêmicos são componentes dos ecossistemas que podem ser consumidos diretamente ou que contribuem para gerar condições adequadas para o bem-estar humano (Quijas et al. 2010). Levantar informações acerca de fragmentos florestais, principalmente aqueles próximos a centros urbanos, é de grande importância uma vez que eles podem ser uma importante fonte de serviços de provimento (alimento, água, plantas medicinais), serviços de regulação (temperaturas mais amenas, proteção contra erosões) e serviços culturais (valores estéticos, educacionais, espirituais) as comunidades próximas (Diraiappah e Naeem 2005).

Desse modo, o objetivo do presente trabalho foi descrever a florística e fitossociologia de um fragmento de floresta estacional semidecídua no agreste paraibano. As informações aqui divulgadas poderão nos oferecer um panorama geral sobre o estado de conservação do fragmento estudado, bem como destacar potenciais serviços ecossistêmicos que são oferecidos a população e podem ser perdidos caso os recursos não sejam utilizados de forma sustentável.

\section{MATERIAL e MÉtodos}

\section{Caracterização da área de estudo}

A área de estudo compreende um fragmento de floresta estacional semidecídua sub-montana (IBGE, 2012), situado em propriedade particular chamada de Fazenda Ipuarana, localizada no município de Lagoa Seca, Paraíba, Brasil as margens da BR-104, próximo ao Km 117, nas coordenadas (79’29’'s; 3552’02”W) (Figura 1).

Figura 1. Fragmento florestal situado na Fazenda Ipuarana, Lagoa Seca - PB. Base de dados: SIRGAS 2000.

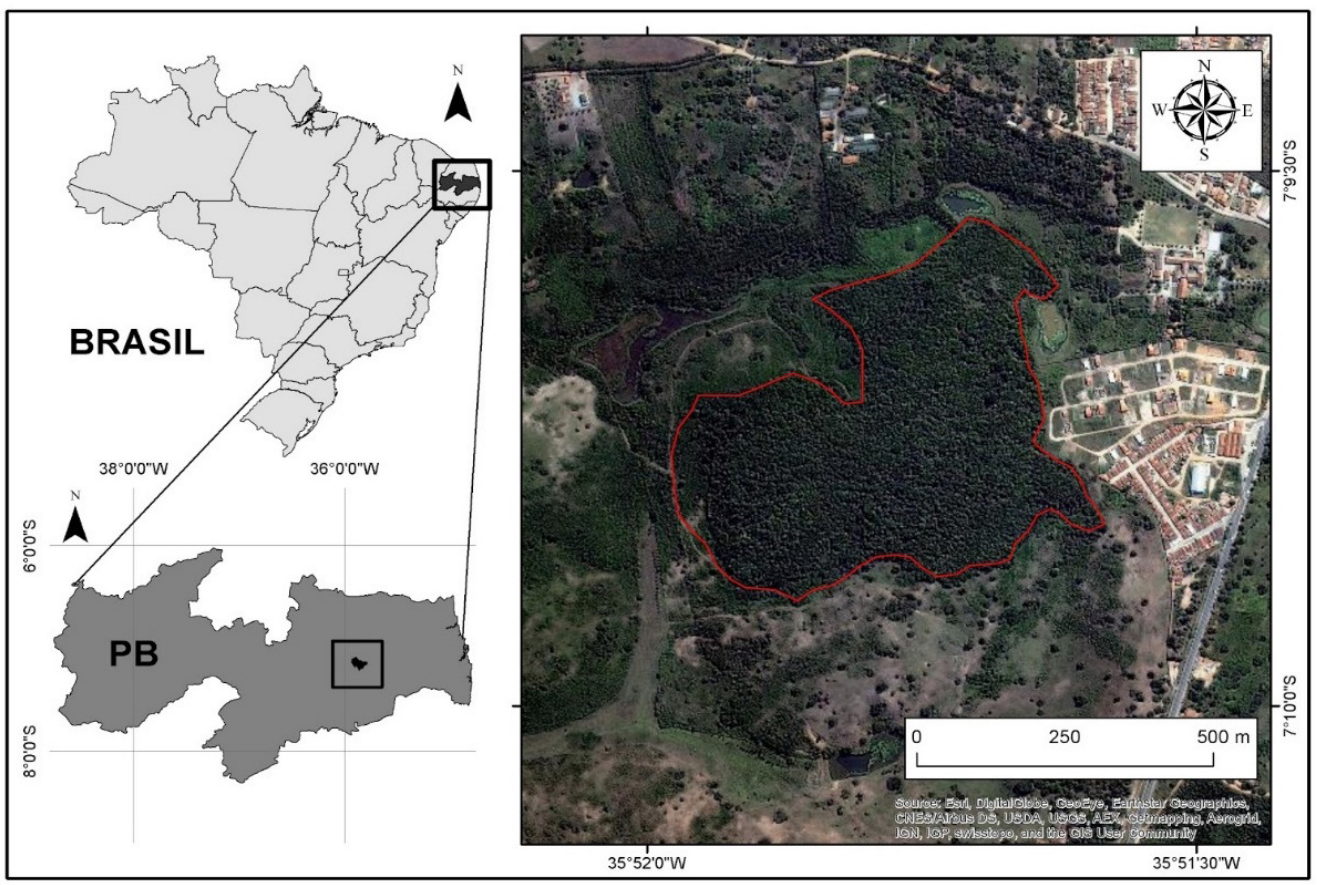


O fragmento foi selecionado devido sua fitofisionomia de transição (Lourenço e Barbosa 2003) entre a caatinga hiperxerófila (precipitação média anual de $500 \mathrm{~mm}$ ) e a floresta atlântica úmida (precipitação anual média superior a $2000 \mathrm{~mm}$ ). Durante as coletas pôde-se perceber que a área sofre constantemente com perturbações de cunho antrópico, sendo estas expressas pela presença de corte seletivo das árvores, além do descarte de resíduos no interior do fragmento. Estas atividades se justificam pela grande proximidade existente entre o perímetro urbano e área de estudo. O fragmento engloba 36 hectares e a precipitação média anual é de aproximadamente $900 \mathrm{~mm}$ (AESA 2019), com uma estação seca de quatro a cinco meses (Andrade et al. 1999). O clima da região é do Tipo As, caracterizado por ser quente e úmido, seguindo a classificação de Köppen-Geiger (Alvares et al. 2014).

\section{Coleta e análise dos dados}

Para amostragem da vegetação foi utilizado o método de parcelas permanentes (Felfili et al. 2005) com todas as árvores incluídas sendo etiquetadas com placas de alumínio em ordem sequencial e cada vértice das parcelas sendo demarcado por uma haste de ferro de aproximadamente $30 \mathrm{~cm}$. Ao total foram alocadas 25 parcelas com dimensões de $20 \times 20 \mathrm{~m}$, totalizando uma área amostral de um hectare. As parcelas foram distribuídas em seis transecções que se estenderam das partes mais altas para as mais baixas do fragmento. As transecções foram estabelecidas em locais que não apresentassem grande proximidade da borda e da trilha principal que corta o fragmento, para que eles não interferissem nos resultados.

Todos os indivíduos arbóreos vivos, com circunferência à altura do peito (CAP) acima de $15 \mathrm{~cm}$ estabelecidos no interior das parcelas, foram mesurados com fita métrica graduada em centímetros e suas respectivas alturas estimadas. Indivíduos que apresentavam caules múltiplos tiveram suas circunferências medidas uma a uma individualmente. Posteriormente, com todos os valores de circunferência anotados, foi calculado o diâmetro de cada indivíduo.

A identificação taxonômica do material botânico coletado foi feita por meio de consultas a especialistas, ao Herbário Manuel Arruda Câmara (HACAM) da Universidade Estadual da Paraíba e ao Herbário Lauro Pires Xavier (JPB) da Universidade Federal da Paraíba. O artigo "Flora da Fazenda Ipuarana, Lagoa Seca, Paraíba (Guia de Campo)" (Lourenço e Barbosa 2003) também foi utilizado para auxiliar na identificação das espécies coletadas, uma vez que o trabalho apresenta a descrição detalhada das espécies presentes na área estudada. A classificação das espécies em famílias seguiu o sistema Angiosperm Phylogeny Group (APG IV 2016).

Para compreensão da estrutura horizontal da comunidade, foram utilizados os parâmetros estruturais de densidade absoluta e relativa, dominância absoluta e relativa e frequência absoluta e relativa, além do valor de importância (VI), além dos valores de área basal por espécie e total, o Índice de Diversidade de Shannon $\left(\mathrm{H}^{\prime}\right)$. Todos esses parâmetros da vegetação foram calculados utilizando o software R versão 3.6. (R Core Team 2019). Ao final foram elaborados histogramas de distribuição de árvores por classes de diâmetro (cm) e altura (m) a partir dos indivíduos levantados.

\section{Resultados E Discussão}

Foram amostrados 1835 indivíduos, pertencentes a 73 espécies e 27 famílias no fragmento estudado. As famílias mais representativas foram Fabaceae (15 espécies), Myrtaceae (10 espécies) e Sapindaceae e Salicaceae (três espécies cada) (Tabela 1). 
Tabela 1. Lista florística das espécies encontradas na área estudada e suas respectivas famílias, área basal $\left(\mathrm{m}^{2} /\right.$ ha), diâmetro médio $(\mathrm{cm})$ e altura média $(\mathrm{m})$.

\begin{tabular}{|c|c|c|c|}
\hline Família Botânica/Espécies & Área basal & Diâmetro médio & Altura média \\
\hline \multicolumn{4}{|l|}{ ANACARDIACEAE } \\
\hline Anacardium occidentale $\mathrm{L}$. & 0,02 & 10,6 & 6,0 \\
\hline \multicolumn{4}{|l|}{ APOCYNACEAE } \\
\hline Aspidosperma parvifolium A. DC. & 0,01 & 12,9 & 10,1 \\
\hline Apocynaceae sp. 1 & 0,20 & & \\
\hline \multicolumn{4}{|l|}{ ARACEAE } \\
\hline Syagrus oleraceae (Mart.) Becc. & 0,56 & 15,6 & 10,1 \\
\hline \multicolumn{4}{|l|}{ BIGNONIACEAE } \\
\hline Handroanthus impetiginosus (Mart. ex DC.) Mattos & 0,40 & 13,2 & 8,5 \\
\hline Handroanthus serratifolius (Vahl) & 2,47 & 32,6 & 14,6 \\
\hline \multicolumn{4}{|l|}{ CAPPARACEAE } \\
\hline Cynophalla flexuosa (L.) J. Presl & 0,31 & 12,4 & 7,2 \\
\hline \multicolumn{4}{|l|}{ CELASTRACEAE } \\
\hline Maytenus rigida Mart. & 0,001 & 4,7 & 2,5 \\
\hline \multicolumn{4}{|l|}{ CLUSIACEAE } \\
\hline Clusia bilariana Schltdl. & 0,67 & 14,6 & 9,7 \\
\hline \multicolumn{4}{|l|}{ CORDIACEAE } \\
\hline Cordia toqueve Aubl. & 0,19 & 14,5 & 10,1 \\
\hline Cordia trichotoma (Vell.) Arráb. ex Steud. & 0,04 & 24,8 & 18,0 \\
\hline \multicolumn{4}{|l|}{ ERYTROXILACEAE } \\
\hline Erythroxylum simonis Plowman & 0,28 & 6,5 & 5,4 \\
\hline Erythroxylum ssp. & 0,03 & 7,2 & 7,0 \\
\hline \multicolumn{4}{|l|}{ EUPHORBIACEAE } \\
\hline Sapium glandulosum (L.) Morong & 0,002 & 5,4 & 5,0 \\
\hline Sebastiania jacobinensis (Müll.Arg.) Müll.Arg & 0,18 & 7,8 & 7,8 \\
\hline \multicolumn{4}{|l|}{ FABACEAE } \\
\hline Albizia inundata (Mart.) Barneby \& J.W.Grimes & 0,49 & 17,1 & 10,3 \\
\hline Albizia polycephala (Benth.) Killip & 0,52 & 14,4 & 11,2 \\
\hline Anadenanthera colubrina (Vell.) Brenan & 0,001 & 8,8 & 9,3 \\
\hline Bowdicbia virgilioides Kunth & 0,49 & 10,1 & 8,2 \\
\hline Cassia ferruginea (Schrader) Schrader ex DC & 0,05 & 12,5 & 9,2 \\
\hline Chloroleucon mangense (Jacq.) Britton \& Rose & 0,49 & 23,1 & 11,3 \\
\hline Dablstedtia araripensis (Benth.) M.J. Silva \& A.M.G. Azevedo & 0,01 & 6,6 & 6,4 \\
\hline Dablstedtia ssp. & 0,07 & 7,1 & 6,7 \\
\hline Fabaceae sp. 1 & 0,02 & 10,5 & 11,3 \\
\hline Fabaceae sp. 2 & 0,05 & 11,0 & 7,0 \\
\hline Gliricidia sepium (Jacq.) Kunth ex Walp. & 0,40 & 17,6 & 11,3 \\
\hline Hymenaea courbaril L. & 0,36 & 10,0 & 7,2 \\
\hline Libidibia ferrea (Mart. ex Tul.) L. P.Queiroz & 0,007 & 9,7 & 7,0 \\
\hline Machaerium birtum (Vell.) Stellfeld & 0,24 & 12,3 & 8,1 \\
\hline Senegalia polyphylla (DC.) Britton \& Rose & 0,33 & 12,8 & 8,5 \\
\hline \multicolumn{4}{|l|}{ LAMIACEAE } \\
\hline Vitex rufescens A. Juss. & 0,01 & 7,4 & 7,6 \\
\hline \multicolumn{4}{|l|}{ LYTHRACEAE } \\
\hline Lafoensia glpptocarpa Koehne & 0,10 & 17,1 & 11,0 \\
\hline
\end{tabular}




\section{Família Botânica/Espécies}

MALPIGHIACEAE

Byrsonima vacciniifolia A.Juss.

Malpighiaceae sp.

MELIACEAE

Trichilia birta $\mathrm{L}$.

Trichilia spp.

MYRTACEAE

Campomanesia aromatica (Aubl.) Griseb.

Campomanesia dichotoma (O. Berg) Mattos

Myrcia splendens (Sw.) DC.

Myrcia tomentosa (Aubl.) DC

Myrtaceae sp. 1

Myrtaceae sp. 2

Plinia cauliflora (Mart.) Kausel

Psidium cattleianum Sabine

Psidium oligospermum Mart. ex DC.

Psidium riparium Mart. ex DC

NICTAGINACEAE

Guapira opposita (Vell.) Reitz

PHYLLANTHACEAE

Margaritaria nobilis L. F.

POLYGONACEAE

Coccoloba mollis Casar.

PRIMULACEAE

Myrsine guianensis (Aubl.) Kuntze

RHAMNACEAE

Ziziphus joazeiro Mart.

RUBIACEAE

Chiococca alba (L.) Hitchc.

Rubiaceae sp.

RUTACEAE

Zanthoxylum petiolare A. St.-Hil. \& Tul

SALICACEAE

Casearia aculeata Jacq.

Casearia sylvestris $\mathrm{Sw}$.

Xylosma prockia (Turcz.) Turcz.

SAPINDACEAE

Allopbylus puberulus (Cambess.) Radlk.

Cupania impressinervia Acev.-Rodr.

Talisia esculenta (A. St.-Hil.) Radlk

SAPOTACEAE

Manilkara salzmannii (A. DC.) H. J. Lam

VOCHYSIACEAE

Callisthene fasciculata Mart.

\section{Área basal Diâmetro médio Altura média}

0,61

11,3

8,2

0,01

9,8

10,5

0,59

11,0

9,9

0,04

6,3

5,0

0,71

10,4

7,2

0,81

14,6

9,0

0,38

9,1

7,1

0,13

8,1

6,0

0,37

9,1

6,7

0,06

8,2

8,3

0,006

6,5

5,0

0,02

8,1

6,0

0,88

9,9

7,9

0,16

7,4

5,4

2,49

12,7

9,4

0,81

12,0

8,7

0,12

11,4

9,1

0,30

15,1

11,3

0,002

5,4

8,0

0,05

15,5

10,1

0,03

11,3

8,6

0,77

14,6

10,5

0,29

9,1

8,3

0,84

18,8

11,0

0,005

5,7

7,0

0,80

8,5

7,7

7,7

1,08

0,25

0,39

12,0

10,5

0,32

14,4

9,0 


\begin{tabular}{lccc}
\hline Família Botânica/Espécies & Área basal & Diâmetro médio & Altura média \\
\hline NÃO IDENTIFICADAS & & & \\
Morfo espécie 1 & 0,52 & 12,2 & 9,5 \\
Morfo espécie 2 & 0,23 & 14,8 & 6,9 \\
Morfo espécie 3 & 0,05 & 10,4 & 9,8 \\
Morfo espécie 4 & 0,01 & 6,1 & 4,8 \\
Morfo espécie 5 & 0,01 & 6,8 & 7,4 \\
Morfo espécie 6 & 0,12 & 39,4 & 16,0 \\
Morfo espécie 7 & 0,02 & 11,0 & 8,0 \\
Morfo espécie 8 & 0,01 & 6,1 & 9,0 \\
Morfo espécie 9 & 0,01 & 11,4 & 10,0 \\
Morfo espécie 10 & 0,003 & 7,0 & 7,0 \\
Morfo espécie 11 & 0,002 & 5,7 & 7,0 \\
\hline
\end{tabular}

Fabaceae e Myrtaceae são responsáveis por 34,24\% de todas as espécies levantadas. Outros estudos desenvolvidos em fragmentos de florestas estacionais no Nordeste também apontaram a família Fabaceae como a mais representativa por sua expressiva riqueza de espécies (Pereira et al. 2002; Andrade e Rodal 2004; Rodal et al. 2005; Nascimento e Rodal 2008; Lopes et al. 2008; Cunha e Silva Júnior 2014). A grande representatividade desse grupo nos fragmentos citados anteriormente pode ser justificada por Fabaceae ser uma das maiores famílias de angiospermas em número de espécies do mundo (Giulietti et al. 2005), além de possuir distribuição cosmopolita (Di Stasi e Hiruma-Lima 2002).

A família Myrtaceae é uma das principais famílias de angiospermas neotropicais (Gentry 1988), sendo uma das 10 famílias com maior riqueza de espécies no Brasil (Sobral et al. 2014), possuindo grande representatividade em ambientes ligados a Floresta Atlântica (Gressler et al. 2006; Salimon e Negrelle 2001). Como o fragmento estudado é caracterizado como uma floresta de transição, cuja fisionomia é semelhante à apresentada pela Floresta Atlântica encontrada próxima ao litoral e os níveis de precipitação estão próximos aos $1000 \mathrm{~mm}$, o elevado número de espécies de Myrtaceae pode estar relacionado a esses fatores.

Tratando-se das espécies amostradas, as que apresentaram maiores valores de densidade absoluta foram: $C$. impressinervia, G. opposita , A. puberulus, P. oligospermum e E. simonis Os maiores valores de VI também pertenceram a cinco espécies: G. opposita (23,24\%), C. impressinervia (18,76\%), A. puberulus (14,35\%), H. serratifolius (14,24\%) e P. oligospermum (12,32\%) (Tabela 2). Apesar de ser uma das espécies com maior número de indivíduos, E. simonis não está entre as cinco de maior VI. Isso pode ser justificado pelos valores relativamente baixos de área basal e dominância relativa apresentados pela espécie quando comparados as espécies de maior VI, indicando que a população de E. simonis é composta de indivíduos de diâmetro reduzido.

Tabela 2. Lista fitossociológica em ordem decrescente de acordo com valor de importância. $\mathrm{NI}=$ número de indivíduos, $\mathrm{DA}=$ densidade absoluta (ind./ha), DR = densidade relativa (ind./m2), FA = frequência absoluta $(\%), F R=$ frequência relativa $(\%), \operatorname{DoA}=$ dominância absoluta $(\%)$, DoR $=$ dominância relativa $(\%), \mathrm{VI}=$ valor de importância (\%).

\begin{tabular}{lccccccc}
\hline Espécies & DA & DR & FA & FR & DoA & DoR & VI \\
\hline Guapira opposita & 154,00 & 8,39 & 92,00 & 4,29 & 2,53 & 10,56 & 23,24 \\
Cupania impressinervia & 206,00 & 11,23 & 64,00 & 2,99 & 1,09 & 4,55 & 18,76 \\
Allophylus puberulus & 123,00 & 6,70 & 92,00 & 4,29 & 0,81 & 3,36 & 14,35 \\
Handroanthus serratifolius & 24,00 & 1,31 & 56,00 & 2,61 & 2,48 & 10,32 & 14,24 \\
Psidium oligospermum & 90,00 & 4,90 & 80,00 & 3,73 & 0,88 & 3,69 & 12,32 \\
Margaritaria nobilis & 59,00 & 3,22 & 68,00 & 3,17 & 0,81 & 3,39 & 9,78 \\
Campomanesia aromática & 67,00 & 3,65 & 52,00 & 2,43 & 0,72 & 3,00 & 9,07
\end{tabular}




\begin{tabular}{|c|c|c|c|c|c|c|c|}
\hline Espécies & DA & DR & FA & FR & DoA & DoR & VI \\
\hline Byrsonima vaccinïfolia & 47,00 & 2,56 & 68,00 & 3,17 & 0,62 & 2,58 & 8,32 \\
\hline Erythroxylum simonis & 73,00 & 3,98 & 64,00 & 2,99 & 0,28 & 1,18 & 8,14 \\
\hline Campomanesia dichotoma & 38,00 & 2,07 & 44,00 & 2,05 & 0,82 & 3,40 & 7,52 \\
\hline Zanthoxylum petiolare & 36,00 & 1,96 & 48,00 & 2,24 & 0,77 & 3,23 & 7,43 \\
\hline Trichilia birta & 52,00 & 2,83 & 44,00 & 2,05 & 0,59 & 2,47 & 7,36 \\
\hline Clusia hilariana & 33,00 & 1,80 & 52,00 & 2,43 & 0,68 & 2,82 & 7,05 \\
\hline Bowdichia virgilioides & 57,00 & 3,11 & 40,00 & 1,87 & 0,49 & 2,06 & 7,03 \\
\hline Syagrus oleraceae & 28,00 & 1,53 & 60,00 & 2,80 & 0,57 & 2,37 & 6,69 \\
\hline Myrtaceae sp. 1 & 51,00 & 2,78 & 48,00 & 2,24 & 0,38 & 1,59 & 6,61 \\
\hline Casearia sylvestris & 22,00 & 1,20 & 36,00 & 1,68 & 0,86 & 3,57 & 6,45 \\
\hline Morfo espécie 1 & 37,00 & 2,02 & 48,00 & 2,24 & 0,52 & 2,18 & 6,43 \\
\hline Myrcia splendens & 49,00 & 2,67 & 44,00 & 2,05 & 0,39 & 1,62 & 6,34 \\
\hline Casearia aculeata & 40,00 & 2,18 & 44,00 & 2,05 & 0,30 & 1,25 & 5,49 \\
\hline Hymenaea courbaril & 40,00 & 2,18 & 36,00 & 1,68 & 0,37 & 1,53 & 5,39 \\
\hline Psidium riparium & 35,00 & 1,91 & 60,00 & 2,80 & 0,16 & 0,67 & 5,38 \\
\hline Manilkara salzmannii & 27,00 & 1,47 & 48,00 & 2,24 & 0,39 & 1,64 & 5,35 \\
\hline Sebastiania jacobinensis & 32,00 & 1,74 & 52,00 & 2,43 & 0,18 & 0,76 & 4,93 \\
\hline Albizia polycephala & 26,00 & 1,42 & 28,00 & 1,31 & 0,53 & 2,20 & 4,92 \\
\hline Senegalia polyphylla & 23,00 & 1,25 & 48,00 & 2,24 & 0,34 & 1,41 & 4,90 \\
\hline Apocynaceae sp. & 40,00 & 2,18 & 40,00 & 1,87 & 0,20 & 0,84 & 4,88 \\
\hline Handroanthus impetiginosus & 20,00 & 1,09 & 40,00 & 1,87 & 0,40 & 1,68 & 4,63 \\
\hline Albizia inundata & 19,00 & 1,04 & 32,00 & 1,49 & 0,50 & 2,08 & 4,61 \\
\hline Cynophalla flexuosa & 21,00 & 1,14 & 40,00 & 1,87 & 0,32 & 1,32 & 4,33 \\
\hline Machaerium birtum & 15,00 & 0,82 & 44,00 & 2,05 & 0,24 & 1,00 & 3,87 \\
\hline Chloroleucon mangense & 8,00 & 0,44 & 24,00 & 1,12 & 0,49 & 2,04 & 3,60 \\
\hline Aspidosperma parvifolium & 18,00 & 0,98 & 28,00 & 1,31 & 0,29 & 1,20 & 3,49 \\
\hline Callisthene fasciculata & 17,00 & 0,93 & 24,00 & 1,12 & 0,33 & 1,37 & 3,42 \\
\hline Gliricidia sepium & 13,00 & 0,71 & 16,00 & 0,75 & 0,40 & 1,67 & 3,13 \\
\hline Myrsine guianensis & 13,00 & 0,71 & 24,00 & 1,12 & 0,30 & 1,25 & 3,08 \\
\hline Morfo espécie 2 & 12,00 & 0,65 & 28,00 & 1,31 & 0,24 & 0,99 & 2,95 \\
\hline Myrcia tomentosa & 23,00 & 1,25 & 24,00 & 1,12 & 0,13 & 0,56 & 2,93 \\
\hline Cordia toqueve & 10,00 & 0,54 & 28,00 & 1,31 & 0,20 & 0,82 & 2,68 \\
\hline Coccoloba mollis & 10,00 & 0,54 & 28,00 & 1,31 & 0,12 & 0,52 & 2,37 \\
\hline Myrtaceae sp. 2 & 17,00 & 0,93 & 16,00 & 0,75 & 0,07 & 0,30 & 1,98 \\
\hline Dablstedtia ssp. & 9,00 & 0,49 & 8,00 & 0,37 & 0,25 & 1,06 & 1,92 \\
\hline Talisia esculenta & 10,00 & 0,54 & 20,00 & 0,93 & 0,06 & 0,25 & 1,72 \\
\hline Erythroxylum spp. & 8,00 & 0,44 & 24,00 & 1,12 & 0,04 & 0,15 & 1,70 \\
\hline Morfo espécie 3 & 6,00 & 0,33 & 20,00 & 0,93 & 0,06 & 0,24 & 1,50 \\
\hline Dablstedtia araripensis & 7,00 & 0,38 & 20,00 & 0,93 & 0,03 & 0,11 & 1,42 \\
\hline Trichilia ssp. & 7,00 & 0,38 & 16,00 & 0,75 & 0,05 & 0,20 & 1,33 \\
\hline Lafoensia glyptocarpa & 4,00 & 0,22 & 8,00 & 0,37 & 0,10 & 0,43 & 1,02 \\
\hline Morfo espécie 4 & 4,00 & 0,22 & 16,00 & 0,75 & 0,01 & 0,05 & 1,02 \\
\hline Cassia ferruginea & 4,00 & 0,22 & 12,00 & 0,56 & 0,05 & 0,22 & 0,99 \\
\hline Morfo espécie 5 & 5,00 & 0,27 & 12,00 & 0,56 & 0,02 & 0,08 & 0,91 \\
\hline Fabaceae sp. 1 & 5,00 & 0,27 & 8,00 & 0,37 & 0,05 & 0,22 & 0,86 \\
\hline Vitex rufescens & 4,00 & 0,22 & 12,00 & 0,56 & 0,02 & 0,08 & 0,85 \\
\hline Anadenanthera colubrina & 3,00 & 0,16 & 12,00 & 0,56 & 0,02 & 0,08 & 0,80 \\
\hline
\end{tabular}




\begin{tabular}{lccccccc}
\hline Espécies & DA & DR & FA & FR & DoA & DoR & VI \\
\hline Chiococca alba & 3,00 & 0,16 & 8,00 & 0,37 & 0,06 & 0,25 & 0,78 \\
Psidium cattleianum & 5,00 & 0,27 & 8,00 & 0,37 & 0,03 & 0,12 & 0,77 \\
Morfo espécie 6 & 1,00 & 0,05 & 4,00 & 0,19 & 0,12 & 0,51 & 0,75 \\
Rubiaceae sp. & 3,00 & 0,16 & 8,00 & 0,37 & 0,03 & 0,13 & 0,66 \\
Fabaceae sp. 2 & 3,00 & 0,16 & 8,00 & 0,37 & 0,03 & 0,12 & 0,65 \\
Morfo espécie 7 & 2,00 & 0,11 & 8,00 & 0,37 & 0,02 & 0,09 & 0,57 \\
Anacardium occidentale & 2,00 & 0,11 & 8,00 & 0,37 & 0,02 & 0,08 & 0,57 \\
Xylosma prockia & 2,00 & 0,11 & 8,00 & 0,37 & 0,01 & 0,02 & 0,50 \\
Cordia trichotoma & 1,00 & 0,05 & 4,00 & 0,19 & 0,05 & 0,20 & 0,44 \\
Malpighiaceae sp. 1 & 2,00 & 0,11 & 4,00 & 0,19 & 0,02 & 0,07 & 0,36 \\
Plinia cauliflora & 2,00 & 0,11 & 4,00 & 0,19 & 0,01 & 0,03 & 0,32 \\
Morfo espécie 8 & 1,00 & 0,05 & 4,00 & 0,19 & 0,01 & 0,05 & 0,29 \\
Morfo espécie 9 & 1,00 & 0,05 & 4,00 & 0,19 & 0,01 & 0,04 & 0,28 \\
Libidibia férrea & 1,00 & 0,05 & 4,00 & 0,19 & 0,01 & 0,03 & 0,27 \\
Morfo espécie 10 & 1,00 & 0,05 & 4,00 & 0,19 & 0,00 & 0,02 & 0,26 \\
Morfo espécie 11 & 1,00 & 0,05 & 4,00 & 0,19 & 0,00 & 0,01 & 0,25 \\
Sapium glandulosum & 1,00 & 0,05 & 4,00 & 0,19 & 0,00 & 0,01 & 0,25 \\
Ziziphus joazeiro & 1,00 & 0,05 & 4,00 & 0,19 & 0,00 & 0,01 & 0,25 \\
Maytenus rígida & 1,00 & 0,05 & 4,00 & 0,19 & 0,00 & 0,01 & 0,25 \\
\hline
\end{tabular}

Guapira opposita foi à espécie de maior VI $(23,54 \%)$ na área de estudo devido aos maiores valores de dominância e frequência relativa e o segundo maior valor de densidade relativa (Figura 2). Essa espécie é amplamente distribuída em florestas estacionais e ombrófilas na região Sul e Sudeste do Brasil, além de também ser uma espécie comum em florestas estacionais e Caatinga na região Nordeste (Sá 2015). A espécie G. opposita também se destacou como uma das espécies de maior VI em ambientes semelhantes no estado Paraíba, podendo ser destacados estudos desenvolvidos na Reserva Ecológica Estadual "Mata do Pau-Ferro" no município de Areia (Andrade et al. 2006) e em um fragmento florestal no município de Bananeiras (Santos et al. 2019). Em um contexto local, essa espécie pode representar um recurso importante para a população que vive no entorno do fragmento, uma vez que sua madeira pode ser utilizada para construções e seus frutos e folhas utilizados para alimentação animal (Melo et. al. 2008; Alves et. al. 2015).

Cupania impressinervia possui o segundo maior VI (18,96\%) com baixa frequência relativa, terceira maior dominância relativa e a maior densidade relativa da comunidade, sendo este o fator determinante na definição do seu VI. Ela também se apresenta como umas das espécies de maior VI no estudo de Santos et al. (2019) desenvolvido no município de Bananeiras. Além disso, Santana et al. (2018) destacam essa espécie como umas das mais abundantes em fragmento de floresta semidecídua pertencente a FLONA de Ibura no estado de Sergipe. Espécies do gênero Cupania geralmente possuem uso medicinal (Alves et al. 2015). Por exemplo, as folhas da espécie Cupania vernalis podem ser utilizadas em infusões que são administradas para tratamento de bronquite (Barata-Silva et al. 2005). A investigação sobre usos de C. impressinervia se faz necessária, uma vez que esta é uma espécie abundante em florestas semidecíduas do Nordeste e espécies muito próximas a ela já são utilizadas como plantas medicinais.

Allophylus puberulus possui o terceiro maior VI (14,68\%) com maior frequência relativa e terceira maior densidade relativa, mostrando que dentre as espécies é a que se encontrou mais dispersa com certa regularidade na comunidade. Andrade et al. (2006) também referiram essa espécie como uma das que apresentam os maiores VIs na Reserva Ecológica Estadual "Mata do Pau-ferro", evidenciando a semelhança das comunidades em relação as espécies de maior importância. Em estudo etnobotânico desenvolvido na comunidade "Vaca Brava", situada 
próxima a "Mata do Pau-Ferro", Abreu et al. (2011) descreveram que a espécie é vista como "não útil” pelos entrevistados. No entanto o conhecimento sobre determinada espécie pode variar entre diferentes localidades, a prova disso é que comunidades próximas a vegetação de restinga no estado do Rio de Janeiro citam $A$. puberulus como uma espécie com bom potencial combustível (Fonseca-Kruel et al. 2009).

Handroanthus serratifolius apresentou o quarto maior VI (14,64\%) com baixa densidade relativa e somente a quinta frequência relativa, porém com segunda maior dominância relativa, mostrando que mesmo estando em baixa densidade a espécie possui indivíduos muito volumosos, com alguns passando dos 60 centímetros de diâmetro, justificando a presença da mesma entre as cinco espécies de maior VI. Em estudo desenvolvido em um fragmento de floresta estacional de terras baixas em Pernambuco, H. serratifolius também se destacou por seu alto VI e principalmente por sua elevada área basal que influenciou diretamente sua alta dominância relativa, como ocorreu no presente estudo (Andrade e Rodal 2004). A casca desta espécie é utilizada para o tratamento de inflamações e doenças do aparelho respiratório (Cordeiro e Félix 2014). Essa característica utilitária aliada a grande beleza de suas flores amarelas, considerada um serviço ecossistêmico cultural, podem ser dois fatores que contribuem com a conservação da espécie na área de estudo, fazendo com que sua população seja composta por indivíduos de grande porte e antigos.

\section{Figura 2. Representação gráfica dos valores de De.R (densidade relativa), Freq.R (frequência relativa) e Dom.R} (dominância relativa) das cinco espécies de maior VI.

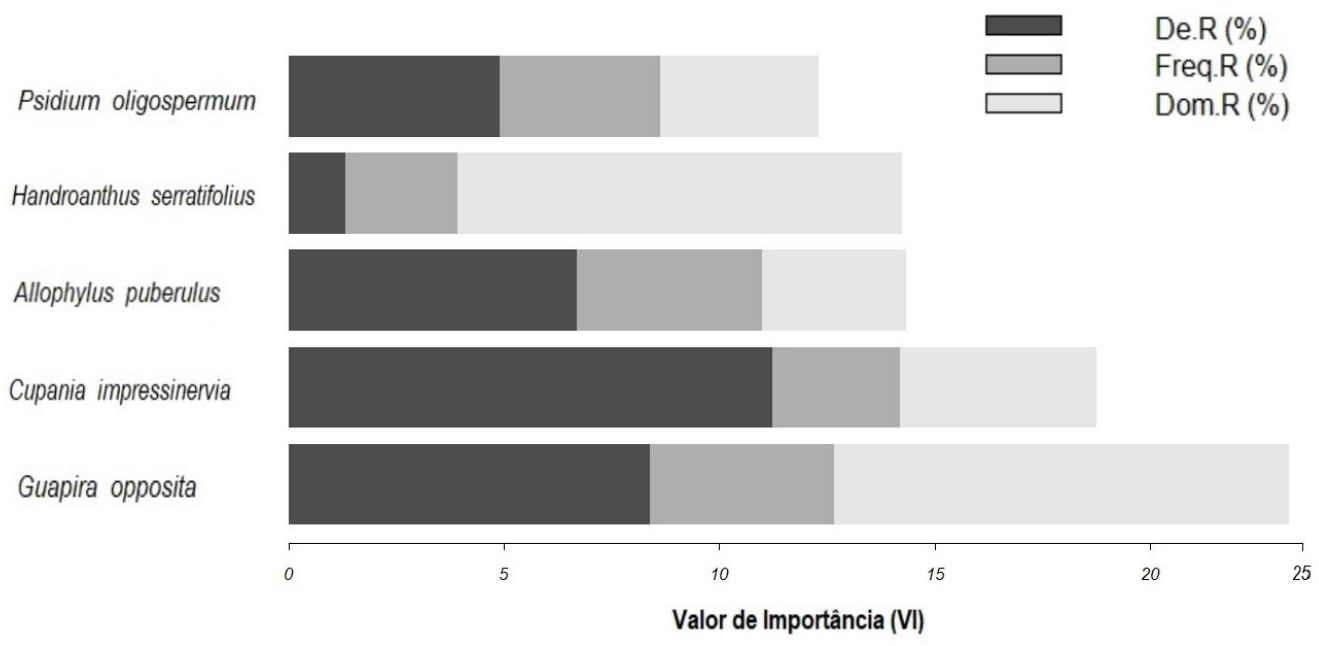

Em termos de diversidade, o fragmento estudado possui maior diversidade de Shannon $\left(H^{\prime}=3,61\right)$ quando comparado a outras florestas estacionais no Nordeste (Pereira et al. 2002; Andrade e Rodal 2004; Andrade et al. 2006; Rodal e Nascimento 2006; Nascimento e Rodal 2008; Cunha et al. 2013), nas quais os valores de diversidade variaram entre 2,72 e 3,42. Essa alta diversidade pode estar relacionada a característica ecotonal do fragmento, uma vez que o mesmo compartilha espécies típicas da Floresta Atlântica e da Caatinga. Além do mais, poucas espécies apresentaram elevadas abundâncias, contribuindo para uma menor dominância ecológica e favorecendo uma maior diversidade. Apesar de ser influenciado pelo esforço amostral e pelo critério de inclusão utilizado no levantamento, o índice de Shannon pode ser uma boa medida para comparar diferentes áreas (Melo 2008).

A área basal total do fragmento é de $23,99 \mathrm{~m}^{2} /$ ha e foi considerada baixa quando comparada a outros fragmentos de floresta estacional semidecidual do Nordeste, como pode ser observado no trabalho de Pereira et al. (2002), Rodal e Nascimento (2006) Nascimento e Rodal (2008), 34,77 $\mathrm{m}^{2} / \mathrm{ha}, 50,32 \mathrm{~m}^{2} / \mathrm{ha}, 39 \mathrm{~m}^{2} / \mathrm{ha}$ respectivamente. Esse resultado evidencia a massiva presença de indivíduos com diâmetro reduzido na área de estudo aqui tratada. Estudos desenvolvidos em áreas semelhantes, como os de Cunha et al. (2013), Andrade et al. (2006) e Andrade e Rodal (2004) obtiveram áreas basais próximas as aqui relatadas, $22,45 \mathrm{~m}^{2} / \mathrm{ha}, 20 \mathrm{~m}^{2} / \mathrm{h}$ e e 24,40 $\mathrm{m}^{2} /$ ha, respectivamente. Todos os referidos autores apontaram que os baixos valores de área basal constatados 
em suas áreas de estudo podem estar relacionados a perturbações de cunho antrópico. Grandes adensamentos de árvores de diâmetro reduzido são reflexos do estágio inicial de regeneração em florestas que passaram por perturbações (Gonzaga et al. 2017).

Verifica-se que a maioria dos indivíduos da área estudada (58,03\%) apresentou diâmetros entre 4,8 e $10 \mathrm{~cm}$ e que $92,02 \%$ deles concentraram-se nas três primeiras classes diamétricas, entre 4,8 e $20 \mathrm{~cm}$ (Figura 3). Assim, pode-se afirmar que na área em questão a distribuição diamétrica dos indivíduos assume a forma de exponencial negativo, com o gráfico assemelhando-se a um J-invertido, indicando que a maior frequência de indivíduos se encontra nas classes de diâmetros reduzidos (Marangon et al. 2008). Na maior classe, acima de $45 \mathrm{~cm}$, apenas oito exemplares foram amostrados. Das espécies, H. serratifolius atingiu o maior valor em relação a diâmetro $(63,66 \mathrm{~cm})$, seguido de L. ferrea $(48,10 \mathrm{~cm})$ e $G$. opposita $(44,10 \mathrm{~cm})$. O diâmetro médio para o remanescente foi de $11,03 \mathrm{~cm}$.

Figura 3. Distribuição de indivíduos por classes diamétricas, com amplitudes de cinco centímetros no fragmento de floresta estacional da Fazenda Ipuarana, Lagoa Seca - PB.

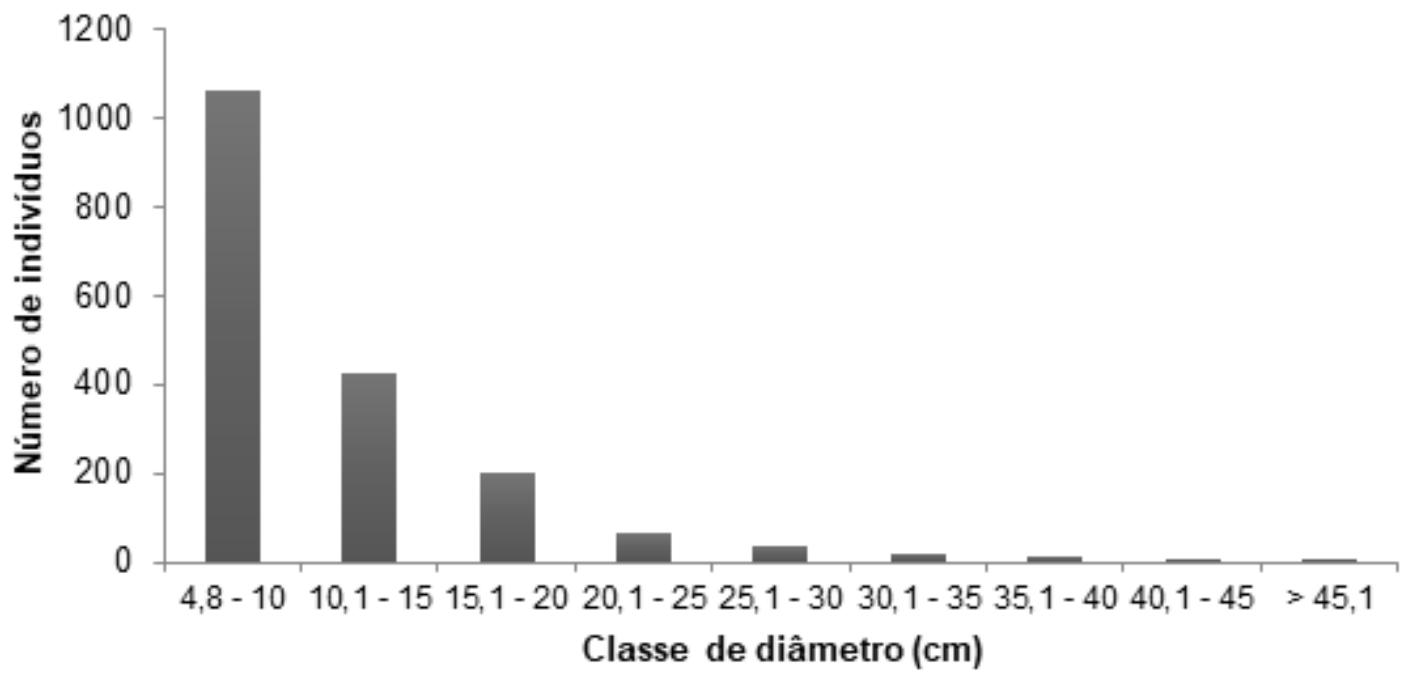

A distribuição hipsométrica manteve um padrão semelhante da diamétrica, em que a maior concentração de indivíduos está nas três primeiras classes de altura (85,39\%), ou seja, até 11 metros de altura (Figura 4). No entanto, a maioria dos indivíduos concentrou-se na segunda classe de altura (44,03\%), entre 5,1 e 8 metros. Poucos foram os indivíduos que atingiram alturas acima de 14 metros (3,05\%). As espécies que atingiram as maiores alturas da comunidade foram M. salzmannii $(19 \mathrm{~m})$, A. polycephala, H. serratifolius e C. trichotoma com 18 metros. A altura média estimada para área foi de 8,23 metros.

Figura 4. Distribuição de indivíduos por classes hipsométricas, com amplitudes de três metros no fragmento de floresta estacional da Fazenda Ipuarana, Lagoa Seca - PB.

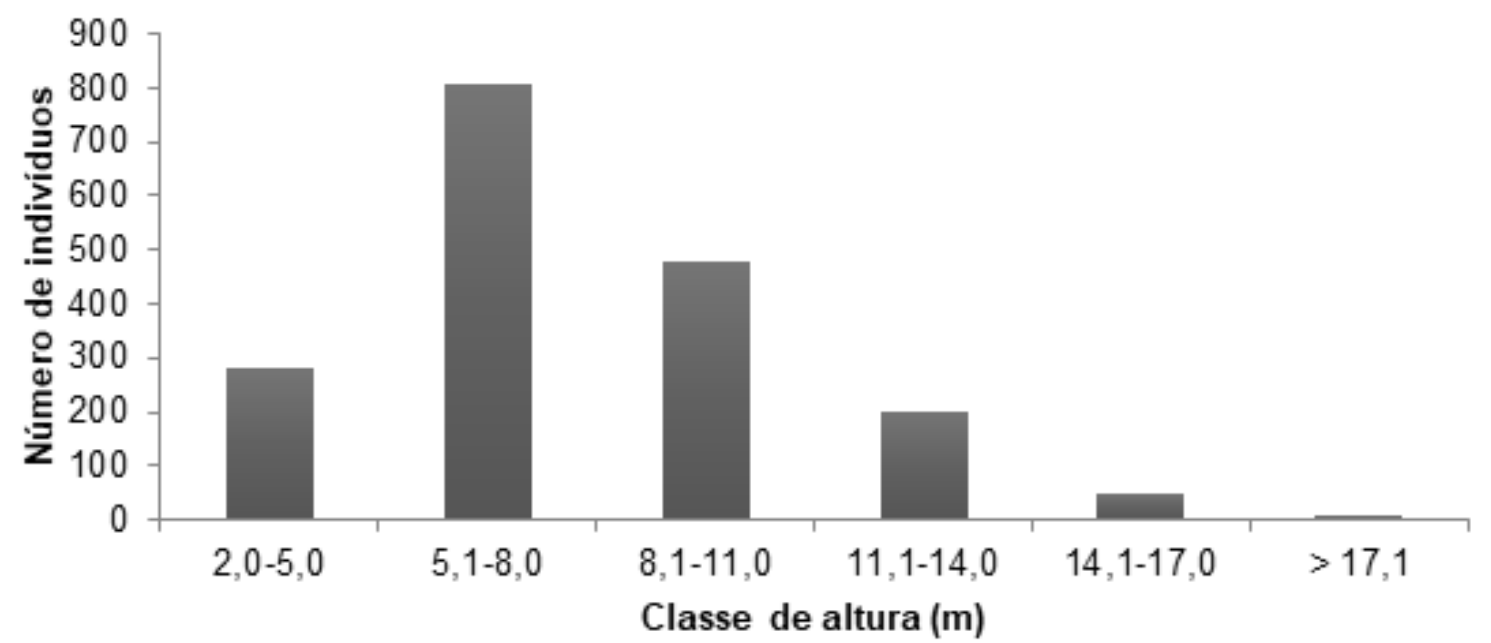




\section{Conclusão}

O fragmento de floresta estacional semidecidual estudado apresentou 76 espécies, das quais, G. opposita, $C$. impressinervia, A. puberulus, $H$. serratifolius e $P$. oligospermum foram as que apresentaram maiores VIs. Apesar de não ser uma unidade de conservação, o fragmento estudado apresentou alta diversidade de espécies $\left(\mathrm{H}^{\prime}=3,61\right) \mathrm{em}$ comparação a outros fragmentos de florestas semidecíduas do Nordeste. No entanto, a área basal total $\left(23,27 \mathrm{~m}^{2} /\right.$ ha) do fragmento foi considerada baixa, situação essa que pode estar relacionada a fatores antrópicos, em razão de o corte seletivo ser constante na área. Nessa perspectiva, a elevada diversidade apresentada por esse fragmento pode estar sendo ameaçada devido a antropização, cenário este que pode estar se repetindo em outros fragmentos de florestas semidecíduas do interior do Nordeste. Esse é um grande risco pois essas florestas podem oferecer serviços ecossistêmicos indispensáveis ao bem-estar das populações que vivem ao entorno delas. O fragmento estudado é uma prova disso, visto que as espécies de maior valor de importância ecológica também podem ser utilizadas para fins utilitaristas, medicinais e estéticos. Além da sua importância ecológica, as florestas representam um bem cultural para as populações que vivem próximas a elas. Nesse sentido, mais levantamentos em florestas estacionais precisam ser realizados para que uma maior quantidade de dados esteja disponível. Esses dados poderão ser utilizados para classificar de forma eficiente esses ambientes e na posterior proposição de estratégias de conservação baseadas nas especificidades dessas florestas.

\section{REFERÊNCIAS}

Abreu DBO, Oliveira Filho RB, Vasconcelos Neto CFA, Lucena CM, Felix LP, Lucena RFP. 2011. Classificação etnobotânica por uma comunidade rural de um brejo de altitude no Nordeste do Brasil. Revista de Biologia e Farmácia (BioFar), 6 (1): 55-74.

AESA. Agência Executiva de Gestão das Águas do Estado da Paraíba. Disponível em: http://www.aesa.pb.gov.br/ aesa-website/.Acesso em: 22 de Jul. 2019.

Alvares CA, Stape JL, Sentelhas PC, Moraes G, Leonardo J, Sparovek G. 2013. Köppen's climate classification map for Brazil. Meteorologische Zeitschrift, 22 (6): 711-728.

Alves JM, Gomes SS, Silva DBS, Rocha PS, Roman AI, Raizer J, Junior VVA, Zefa ZV. 2015. Uso Múltiplo de Espécies Arbóreas Nativas do Fragmento de Floresta Semidecidual Ribeirinha da Fazenda Experimental da Universidade Federal da Grande Dourados. Cadernos de Agroecologia, 9(4): 1-10.

Andrade LA, Reis MG, Reis GG. 1999. Classificação ecológica do Estado da Paraíba. Interpolação de dados climáticos por aproximação numérica. Revista Árvore, 23 (1): 3-32.

Andrade KVSA, Rodal MJN. 2004. Fisionomia e estrutura de um remanescente de floresta estacional semidecidual de terras baixas no nordeste do Brasil. Revista Brasileira de Botânica, 27 (3): 463-474.

Andrade LA, Oliveira FX, Nascimento IS, Fabricante JR, Sampaio EV, Barbosa MR. 2006. Análise florística e estrutural de matas ciliares ocorrentes em brejo de altitude no município de Areia, Paraíba. Revista Brasileira de Ciências Agrárias, 1 (1): 31-40.

APG IV. 2016. An update of the Angiosperm Phylogeny Group classification for the orders and families of flowering plants: APG IV. Botanical Journal of the Linnean Society, 181 (1): 1-20. 
Barata-Silva AW, Macedo RLG, Gomes JE. 2005. Potencial de utilização de espécies arbóreas medicinais no Rio Grande do Sul. Revista Científica Eletrônica de Engenharia Florestal. 3 (6): 1-6.

Cordeiro JMP, Félix LP. 2014. Conhecimento botânico medicinal sobre espécies vegetais nativas da caatinga e plantas espontâneas no agreste da Paraíba, Brasil. Revista Brasileira de Plantas Medicinais, 16 (3 suppl 1): 685-692.

Cunha MDCL, Silva Júnior MC. 2014. Flora e estrutura de Floresta Estacional Semidecidual Montana nos estados da Paraíba e Pernambuco. Nativa, 2 (2): 95-102.

Cunha MDCL, Silva Júnior MCD, Lima RBD. 2013. Fitossociologia do Estrato Lenhoso de uma floresta estacional semidecidual montana na Paraíba, Brasil. Cerne, 19 (2): 271-280.

Davis CC, Anderson WR. 2010. Uma filogenia genérica completa de Malpighiaceae inferida a partir de dados de seqüência de nucleotídeos e morfologia. American Journal of Botany, 97 (12): 2031-2048.

Dexter KG, Pennington RT, Oliveira-Filho AT, Bueno ML, Miranda PLS, Neves DM. 2018. Inserting tropical dry forests into the discussion on biome transitions in the tropics. Frontiers in Ecology and Evolution, 6 (1): 104.

Di Stasi LC, Hiruma-Lima, CA. 2002. Plantas medicinais na Amazônia e na Mata Atlântica. Editora Unesp, São Paulo, p. 604.

Diraiappah AK, Naeem S. 2005. Millennium ecosystem assessment: ecosystems and human well-being: biodiversity synthesis. World Resources Institute, Washington (DC).

Felfili JM, Carvalho FA, Haidar RF. 2005. Manual para o monitoramento de parcelas permanentes nos biomas Cerrado e Pantanal. EdUnB, Brasília, p.55.

Fonseca-Kruel VSD, Araujo DSDD, Sá CFCD, Peixoto AL. (2009). Quantitative ethnobotany of a restinga forest fragment in Rio de Janeiro, Brazil. Rodriguésia, 60 (1): 187-202.

Gentry A. 1988. Changes in plant community Diversity and Florístic composition on environmental and geographical gradients. Annals of the Missouri Botanical Garden, 75 (1): 1-34.

Gressler E, Pizo MA, Morellato LPC. 2006. Polinização e dispersão de sementes em Myrtaceae do Brasil. Brazilian Journal of Botany, 29 (4): 509-530.

Giulietti AM, Harley RM, Queiroz LP, Wanderley MG, Berg CVB. 2005. Biodiversidade e conservação das plantas no Brasil. Megadiversidade, 1: 52-60.

Gonzaga APD, Machado ELM, Felfili JM, Pinto JRR. 2017. Brazilian Decidual Tropical Forest enclaves: floristic, structural and environmental variations. Brazilian Journal of Botany, 40 (2): 417-426.

IBGE - INSTITUTO BRASILEIRO DE GEOGRAFIA E ESTATÍSTICA. 2012. Manual Técnico da Vegetação Brasileira. Rio de Janeiro, p. 271.

Janzen, D. H. 1988. Management of Habitat Fragments in a Tropical Dry Forest: Growth. Annals of the Missouri Botanical Garden, 75(1): 105-116.

Lins RC. 1989. Áreas de exceção do Agreste de Pernambuco. Recife: Sudene, 1: 1-26. 
Lopes CGR, Ferraz EMN, Araújo EL. 2008. Physiognomic-structural characterization of dry-and humid-forest fragments (Atlantic Coastal Forest) in Pernambuco State, NE Brazil. Plant Ecology, 198 (1): 1-18.

Lourenço CEL, Barbosa MRV. 2003. Flora da fazenda Ipuarana, Lagoa Seca, Paraíba (guia de campo). Revista Nordestina de Biologia, 17(1/2): 23-58.

Medeiros H, Forzza RC, Acevedo-Rodríguez P. 2016. Wild relatives of guaraná (Paullinia cupana, Sapindaceae) in southwestern Brazilian Amazon. Systematic Botany, 41(1): 202-228.

Melo AS. 2008. O que ganhamos "confundindo" riqueza de espécies e equabilidade em um índice de diversidade?. Biota Neotropica. 8(3): 21-27.

Melo S, Lacerda VD, Hanazaki N. 2008. Espécies de restinga conhecidas pela comunidade do Pântano do sul, Florianópolis, Santa Catarina, Brasil. Rodriguésia, 59(4), 799-812.

Miranda PLS, Oliveira-Filho AT, Pennington RT, Neves DM, Baker TR, Dexter KG. 2018. Using tree species inventories to map biomes and assess their climatic overlaps in lowland tropical South America. Global ecology and biogeography, 27 (8): 899-912.

Murphy PG, Lugo A. 1986. Ecology of tropical dry forest. Annual Review Ecology and Systematics, 17 (1): 67-88.

Nascimento LD, Rodal MJN. 2008. Fisionomia e estrutura de uma floresta estacional montana do maciço da Borborema, Pernambuco-Brasil. Revista Brasileira de Botânica, 31 (1): 27-39.

Oliveira-Filho AT, Ratter JA. 1995. A study of the origin of central Brazilian forests by the analysis of plant species distribution patterns. Edinburgh Journal of Botany, 52(2): 141-194.

Pennington RT, Lehmann CER, Rowland LM. 2018. Tropical savanas e florestas secas. Current Biology, 28 (9): 541-545.

Pennington RT, Prado DE, Pendry CA. 2000. Neotropical seasonally dry forests and Quaternary vegetation changes. Journal of Biogeography, 27 (2): 261-273.

Pereira IM, Andrade LAD, Barbosa MRDV, Sampaio E. 2002. Composição florística e análise fitossociológica do componente arbustivo-arbóreo de um remanescente florestal no agreste paraibano. Acta Botanica Brasilica, 16 (3): 357-369.

Quijas S, Schmid B, Balvanera P. 2010. Plant diversity enhances provision of ecosystem services: a new synthesis. Basic and Applied Ecology, 11 (7), 582-593.

R Core Team. 2019. R: A language and environment for statistical computing. R Foundation for Statistical Computing, Vienna, Austria. https://www.r-project.org/.

Rodal MJN, Barbosa MRV, Thomas WW. 2008. Do the seasonal forests in northeastern Brazil represent a single floristic unit?. Brazilian Journal of Biology, 68 (3): 467-475.

Rodal MJN, Lucena MDFA, Andrade KVSA, Melo AD. 2005. Mata do Toró: uma floresta estacional semidecidual de terras baixas no nordeste do Brasil. Hoehnea, 32 (2): 283-294.

Rodal MJN, Nascimento LD. 2002. Levantamento florístico da floresta serrana da reserva biológica de Serra Negra, microrregião de Itaparica, Pernambuco, Brasil. Acta Botanica Brasilica, 16 (4), 481-500. 
Rodal MJN, Nascimento LD. 2006. The arboreal component of a dry forest in northeastern Brazil. Brazilian Journal of Biology, 66 (2A): 479-491.

Sá CFC. 2015. Nyctaginaceae. In Lista de Espécies da Flora do Brasil. Jardim Botânico do Rio de Janeiro. Disponível em: < http://floradobrasil.jbrj.gov.br/jabot/floradobrasil/FB10913>. Acesso em: 05 Jul. 2019.

Salimon CI, Negrelle RRB. 2001. Natural Regeneration in a Quaternary Coastal Plain in Southern Brazilian Atlantic Rain Forest. Brazilian Archives of Biology and Technology, 44(2):155-163.

Santana JP, Rocha PA, Silva EVO, Prata APN, Ribeiro AS. 2018. Phenological patterns of zoochoric shrub and tree species in two areas of Ibura National Forest, Northeastern Brazil. Neotropical Biology and Conservation, 13(3): 212-223.

Santos JNB, Medeiros RLS, Santos TED, Barros AP, Oliveira EJBL, Fernande FAS, Wanderley MJA, Barbosa AS. 2018. Diversity and Structure of Adult and Regenerating Arbor Component in Forest 'Submontana', Paraíba-Brazil. Journal of Experimental Agriculture International, 25 (5): 1-14.

Sobral M, Proença C, Souza M, Mazine, F, Lucas E. 2015. Myrtaceae. In Lista de Espécies da Flora do Brasil. Jardim Botânico do Rio de Janeiro. Disponível em: < http://floradobrasil.jbrj.gov.br/jabot/floradobrasil/FB171>. Acesso em: 05 Jul. 2019.

Tabarelli M, Santos AMM. 2004. Uma breve descrição sobre a história natural dos brejos nordestinos. In: Pôrto KC, Cabral JJP, Tabarelli M. (Eds). Brejos de Altitude em Pernambuco e Paraíba, História Natural, Ecologia e Conservação. Brasília, MMA, p. 229-254. 